University of Nebraska - Lincoln

DigitalCommons@University of Nebraska - Lincoln

October 2006

\title{
Application of Coulomb wave function discrete variable representation to atomic systems in strong laser fields
}

\author{
Liang-You Peng \\ University of Nebraska - Lincoln, lypeng@unlserve.unl.edu \\ Anthony F. Starace \\ University of Nebraska-Lincoln, astarace1@unl.edu
}

Follow this and additional works at: https://digitalcommons.unl.edu/physicsstarace

Part of the Physics Commons

Peng, Liang-You and Starace, Anthony F., "Application of Coulomb wave function discrete variable representation to atomic systems in strong laser fields" (2006). Anthony F. Starace Publications. 99. https://digitalcommons.unl.edu/physicsstarace/99

This Article is brought to you for free and open access by the Research Papers in Physics and Astronomy at DigitalCommons@University of Nebraska - Lincoln. It has been accepted for inclusion in Anthony F. Starace Publications by an authorized administrator of DigitalCommons@University of Nebraska - Lincoln. 


\title{
Application of Coulomb wave function discrete variable representation to atomic systems in strong laser fields
}

\author{
Liang-You Peng ${ }^{\text {a) }}$ and Anthony F. Starace \\ Department of Physics and Astronomy, The University of Nebraska-Lincoln, Nebraska 68588-0111
}

(Received 20 July 2006; accepted 5 September 2006; published online 19 October 2006)

\begin{abstract}
We present an efficient and accurate grid method for solving the time-dependent Schrödinger equation for an atomic system interacting with an intense laser pulse. Instead of the usual finite difference (FD) method, the radial coordinate is discretized using the discrete variable representation (DVR) constructed from Coulomb wave functions. For an accurate description of the ionization dynamics of atomic systems, the Coulomb wave function discrete variable representation (CWDVR) method needs three to ten times fewer grid points than the FD method. The resultant grid points of the CWDVR are distributed unevenly so that one has a finer grid near the origin and a coarser one at larger distances. The other important advantage of the CWDVR method is that it treats the Coulomb singularity accurately and gives a good representation of continuum wave functions. The time propagation of the wave function is implemented using the well-known Arnoldi method. As examples, the present method is applied to multiphoton ionization of both the $\mathrm{H}$ atom and the $\mathrm{H}^{-}$ion in intense laser fields. The short-time excitation and ionization dynamics of $\mathrm{H}$ by an abruptly introduced static electric field is also investigated. For a wide range of field parameters, ionization rates calculated using the present method are in excellent agreement with those from other accurate theoretical calculations. (C) 2006 American Institute of Physics.
\end{abstract}

[DOI: $10.1063 / 1.2358351]$

\section{INTRODUCTION}

With the rapid advance of modern laser technologies, lasers of various frequencies and different intensities are routinely available in many laboratories. ${ }^{1}$ Studies of the highly nonlinear interaction of matter with strong laser pulses have revealed many interesting features of physical and chemical processes. ${ }^{2}$ New technologies based on these features are under rapid development and new frontiers of science have been opened. ${ }^{3}$ Newly developed light sources have, for the first time, enabled physicists and chemists to trace and image the electronic motion within atoms and molecules on an attosecond time scale. ${ }^{4}$

However, the multiple reaction paths and the many-body nature of these highly nonlinear and ultrafast processes contribute to the complexities of theoretical interpretations of experimental observations. The high intensities of the applied lasers make perturbation theory no longer applicable. It is necessary to treat the Coulomb interaction and the interaction with the laser field on an equal footing. Many theoretical methods have been designed to describe different kinds of phenomena. To mention just a few, these methods range from the strong field approximation, ${ }^{5}$ the intense-field many-body $S$-matrix theory, ${ }^{6}$ the $R$-matrix Floquet method, ${ }^{7}$ the generalized Floquet theory, ${ }^{8}$ the time-dependent density functional theory, ${ }^{9}$ and the direct numerical integration of the time-dependent Schrödinger equation. ${ }^{10-13}$ Compared to other methods, the direct solution of the time-dependent Schrödinger equation (TDSE) has proved to be very versatile

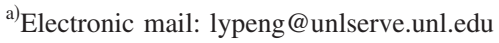

and fruitful in explaining and predicting many experimental measurements for a wide range of laser parameters. Especially when the laser pulse length approaches the few-cycle or subfemtosecond regime, the numerical solution of the TDSE becomes even more appropriate and efficient.

Nevertheless, the accurate integration of the multidimensional TDSE is very computationally demanding. Even the most powerful supercomputer nowadays has only made the $a b$ initio integration of the two-electron atom possible. ${ }^{14}$ Therefore, many approximate theoretical models (such as reduced-dimension models and soft Coulomb potential models) have played an important role in understanding some of the main physical mechanisms underlying strong field phenomena. ${ }^{15}$ On the other hand, it is questionable to use these models to simulate experiments quantitatively. In particular, the dynamical motion in three dimensions is not merely a trivial extension of what happens in two dimensions. ${ }^{16}$ Also, the physics of model systems using the soft Coulomb potential is very sensitive to the softening parameters. ${ }^{17}$ Therefore, in order to produce quantitatively correct results, it is necessary to solve the TDSE in its full dimensionality and to use the real Coulomb potential, with its singularity treated properly. ${ }^{18}$ This is especially crucial when the singularity plays an important role in the problem at hand. ${ }^{16,19}$

In the present paper, we present an accurate and efficient method to solve the TDSE for multiphoton ionization of a hydrogenic atom or ion. Unlike the usual finite difference (FD) discretization of the radial coordinate, ${ }^{10,11}$ the present method discretizes the radial coordinate using the discrete variable representation (DVR) constructed from the positive 
energy Coulomb wave function. We show that the Coulomb wave function DVR (CWDVR) is able to treat the Coulomb singularity naturally and provide a good representation of continuum wave functions. The other advantage of the CWDVR is that it needs three to ten times fewer grid points than the FD method because of the uneven distribution of the grid points: one has a coarser grid at larger distances, where the Coulomb potential plays a less important role and the wave functions oscillate less rapidly. Because the CWDVR is economical, it is a promising step towards a more efficient treatment of many-electron systems, which are extremely computationally demanding. ${ }^{14}$ In addition, many strong field processes, such as above threshold ionization (ATI), highorder harmonic generation (HHG), dynamical stabilization, etc., can be well understood within a single active electron (SAE) picture. Therefore, the hydrogenic atom or ion system serves as a prototype for spherically symmetric atomic systems interacting with intense laser fields.

The rest of this paper is organized as follows. In Sec. II, after a brief introduction to the general DVR method, we provide details of the CWDVR. In Sec. III we apply the CWDVR to discretize the TDSE for one-electron atomic systems in intense laser fields. In Sec. IV, we present some results for multiphoton ionization of $\mathrm{H}$ and $\mathrm{H}^{-}$as well as for the short-time dynamics of $\mathrm{H}$ in a static electric field. We show that ionization rates calculated by the present economical and accurate method are in excellent agreement with other accurate theoretical calculations. Finally, in Sec. V, we present some conclusions. Atomic units (a.u.) are used throughout this paper unless otherwise specified.

\section{COULOMB WAVE FUNCTION DVR}

The DVR method has its origin in the transformation method devised by Harris et al. ${ }^{20}$ for calculating matrix elements of complicated potential functions within a truncated basis set. It was further developed by Dickinson and Certain, ${ }^{21}$ who showed the relationship between the transformation method and the Gaussian quadrature rule for orthogonal polynomials. Light and co-workers ${ }^{22}$ first explicitly used the DVR method as a basis representation for quantum problems rather than as just a means of evaluating Hamiltonian matrix elements. Ever since then, different types of DVR methods have found wide applications in different fields of physical and chemical problems. ${ }^{23}$ There continue to be many efforts to construct new types of DVRs and to apply DVRs in combination with other numerical methods. ${ }^{24}$

Essentially, the DVR method is a representation whose associated basis functions are localized about discrete values of the coordinate under consideration. The DVR greatly simplifies the evaluation of Hamiltonian matrix elements. The potential matrix elements involve merely the evaluation of the interaction potential at the DVR grid points; no integration is needed. The kinetic energy matrix elements can also be calculated very simply (and analytically in most cases). ${ }^{25}$ In this section, we first give a short introduction to the DVR constructed from orthogonal polynomials. Then we present how one constructs the DVR from Coulomb wave functions, which will be used to solve the TDSE of atomic systems in intense laser fields in Sec. III.

\section{A. DVR using orthogonal polynomials}

The DVR basis functions can be constructed from any complete set of orthogonal polynomials, $P_{N}(x),{ }^{25}$ defined in the domain $(a, b)$ with the corresponding weight function $\omega(x)$. Let

$$
\mathcal{P}_{N}(x)=\sqrt{\omega(x) / h_{N}} P_{N}(x),
$$

where $h_{N}$ is a normalization constant chosen so that

$$
\int_{a}^{b} d x \mathcal{P}_{M}(x) \mathcal{P}_{N}(x)=\delta_{M N} .
$$

Then the cardinal function $\mathcal{C}_{i}(x)$ of $\mathcal{P}_{N}(x)$ is given by ${ }^{26}$

$$
\mathcal{C}_{i}(x)=\frac{1}{\mathcal{P}_{N}^{\prime}\left(x_{i}\right)} \frac{\mathcal{P}_{N}(x)}{x-x_{i}},
$$

where the points $x_{i}(i=1,2, \ldots, N)$ are the zeros of $P_{N}(x)$, and $\mathcal{P}_{N}^{\prime}\left(x_{i}\right)$ stands for the first derivative of $\mathcal{P}_{N}(x)$ at $x_{i}$. Clearly, $\mathcal{C}_{i}(x)$ satisfies the cardinality condition

$$
\mathcal{C}_{i}\left(x_{j}\right)=\delta_{i j}
$$

The DVR basis function $f_{i}(x)$ is constructed from the cardinal function $\mathcal{C}_{i}(x)$ as follows:

$$
f_{i}(x)=\frac{1}{\sqrt{\omega_{i}}} \mathcal{C}_{i}(x)
$$

which, at the point $x_{j}$, gives

$$
f_{i}\left(x_{j}\right)=\frac{1}{\sqrt{\omega_{i}}} \delta_{i j} .
$$

We know that the integration of any function $F(x)$ can be calculated using an appropriate quadrature rule associated with the zeros of the orthogonal polynomial, i.e.,

$$
\int_{a}^{b} d x F(x) \simeq \sum_{i=1}^{N} \omega_{i} F\left(x_{i}\right)
$$

where $\omega_{i}$ is the corresponding weight at the point $x_{i}$. From the theory of classical orthogonal polynomials, the integration formula (7) is exact as long as the function $F(x)$ can be expressed as a polynomial of order $2 N-1$ (or lower) times the weight function $\omega(x)$ (cf. Theorem 3.4.1 of Ref. 27). With the help of Eqs. (1), (3), and (5), it is easy to show that the function $f_{i}^{*}(x) f_{j}(x)$ satisfies this condition. Therefore, the following integration can be carried out exactly:

$$
\int_{a}^{b} d x f_{i}^{*}(x) f_{j}(x)=\sum_{k=1}^{N} \omega_{k} f_{i}^{*}\left(x_{k}\right) f_{j}\left(x_{k}\right)=\delta_{i j} .
$$

As a result of Eqs. (6) and (7), any local operator $V(x)$ has a diagonal representation in the DVR basis set, 


$$
\int_{a}^{b} d x f_{i}^{*}(x) V(x) f_{j}(x) \simeq \sum_{k=1}^{N} \omega_{k} f_{i}^{*}\left(x_{k}\right) V\left(x_{k}\right) f_{j}\left(x_{k}\right)=V\left(x_{i}\right) \delta_{i j} .
$$

On the contrary, the representation of a differential operator in the DVR basis is usually a full matrix. Nevertheless, in most cases, the matrix elements of the first and second differential operators,

$$
\int_{a}^{b} d x f_{i}^{*}(x) \frac{d}{d x} f_{j}(x)
$$

and

$$
\int_{a}^{b} d x f_{i}^{*}(x) \frac{d^{2}}{d x^{2}} f_{j}(x)
$$

can be evaluated analytically using the quadrature rule (7). Usually, the final results are very simple expressions of the zeros $x_{i}$ and the number of zeros $N .^{25}$

\section{B. DVR constructed from Coulomb wave functions}

It is well known that a Gaussian quadrature can also be constructed using nonclassical polynomials ${ }^{28}$ and even rational function $\mathrm{s}^{29}$ by means of the standard three-term recursion relations. ${ }^{27}$ A general method for constructing different types of DVRs in this way has been given in Ref. 30. A new type of DVR derived from the usual Legendre DVR has been shown by Machtoub and Zhang ${ }^{31}$ to provide very accurate results for the metastable states of the exotic helium atom, $\bar{p} \mathrm{He}^{+}$.

An appropriate quadrature rule for the Coulomb wave function was given by Dunseath et $a .^{32}$ with explicit expressions for the weights $\omega_{i}$. Therefore, the DVR basis function $f_{i}(r)$ may be similarly constructed from a Coulomb wave function, which satisfies the differential equation [see Eq. (14.1.1) of Ref. 33],

$$
\frac{d^{2}}{d \widetilde{r}^{2}} v(\widetilde{r})+\left[1-\frac{2 \eta}{\widetilde{r}}-\frac{L(L+1)}{\widetilde{r}^{2}}\right] v(\widetilde{r})=0,
$$

where $\eta$ is real and $L$ is a non-negative integer. Equation (12) has a regular solution, $F_{L}(\eta, \widetilde{r})$.

For our purposes, we consider the regular solution for $L=0$ and $\eta<0$. Denoting

$$
\eta=-\frac{Z}{\sqrt{2 E}}
$$

and

$$
\widetilde{r}=r \sqrt{2 E},
$$

we may write Eq. (12) alternatively as

$$
\frac{d^{2}}{d r^{2}} v(r)=-\left[2 E+\frac{2 Z}{r}\right] v(r) \equiv W(r) v(r),
$$

whose solution is given by

$$
v(r)=F_{0}(-Z / \sqrt{2 E}, r \sqrt{2 E}) .
$$

For any given energy $E$ and nuclear charge $Z$, the solution (15) has simple zeros over $(0, \infty)$ (see Fig. 14.3 of Ref. 33). Similarly to Eq. (3), we can define the cardinal function of $v(r)$ as

$$
C_{i}(r)=\frac{1}{v^{\prime}\left(r_{i}\right)} \frac{v(r)}{r-r_{i}},
$$

where $r_{i}$ is the $i$ th zero of $v(r)$ and $v^{\prime}\left(r_{i}\right)$ stands for its first derivative at $r_{i}$. In analogy to Eq. (5), one can construct the Coulomb wave function DVR basis function,

$$
f_{i}(r)=\frac{1}{\sqrt{\omega_{i}}} C_{i}(r)=\frac{1}{\sqrt{\omega_{i}}} \frac{1}{v^{\prime}\left(r_{i}\right)} \frac{v(r)}{r-r_{i}},
$$

which, at the zero $r_{j}$, becomes

$$
f_{i}\left(r_{j}\right)=\frac{1}{\sqrt{\omega_{i}}} \delta_{i j}
$$

Following Schwartz, ${ }^{34}$ Dunseath et al..$^{32}$ constructed an appropriate quadrature rule associated with the zeros $r_{i}$ of the Coulomb wave function in Eq. (15). Using this quadrature rule, one can evaluate the integration of a function $F(r)$ over $(0, \infty)$ as follows:

$$
\int_{0}^{\infty} d r F(r) \simeq \sum_{i=1}^{N} \omega_{i} F\left(r_{i}\right)
$$

where the weight $\omega_{i}$ is given by ${ }^{32}$

$$
\omega_{i} \simeq \frac{\pi}{a_{i}^{2}}
$$

with

$$
a_{i} \equiv v^{\prime}\left(r_{i}\right) .
$$

Using the quadrature rule, Eq. (19), one may show the orthonormality of the CWDVR basis functions,

$$
\int_{0}^{\infty} f_{i}^{*}(r) f_{j}(r) d r \simeq \sum_{k=1}^{N} \omega_{k} f_{i}^{*}\left(r_{k}\right) f_{j}\left(r_{k}\right)=\delta_{i j} .
$$

One may also evaluate matrix elements of the forms in Eqs. (10) and (11) using the same quadrature rule,

$$
\begin{aligned}
P_{i j} & \equiv \int_{0}^{\infty} f_{i}^{*}(r) \frac{d}{d r} f_{j}(r) d r, \\
& \simeq \sum_{k=1}^{N} \omega_{k} f_{i}^{*}\left(r_{k}\right) f_{j}^{\prime}\left(r_{k}\right)=\sum_{k=1}^{N} \frac{\omega_{k}}{\sqrt{\omega_{i} \omega_{j}}} \delta_{i k} C_{j}^{\prime}\left(r_{k}\right),
\end{aligned}
$$

and 


$$
\begin{aligned}
T_{i j} & \equiv-\frac{1}{2} \int_{0}^{\infty} f_{i}^{*}(r) \frac{d^{2}}{d r^{2}} f_{j}(r) d r \\
& \simeq-\frac{1}{2} \sum_{k=1}^{N} \omega_{k} f_{i}^{*}\left(r_{k}\right) f_{j}^{\prime \prime}\left(r_{k}\right)=-\frac{1}{2} \sum_{k=1}^{N} \frac{\omega_{k}}{\sqrt{\omega_{i} \omega_{j}}} \delta_{i k} C_{j}^{\prime \prime}\left(r_{k}\right) .
\end{aligned}
$$

It is easy to show that, for the solution $v(r)$ to Eq. (14), the first and second derivatives of the cardinal function, Eq. (16), are given, respectively, by ${ }^{32,34}$

$$
C_{j}^{\prime}\left(r_{k}\right)=\left(1-\delta_{j k}\right) \frac{a_{k}}{a_{j}} \frac{1}{r_{k}-r_{j}}
$$

and

$$
C_{j}^{\prime \prime}\left(r_{k}\right)=\delta_{j k} \frac{c_{k}}{3 a_{k}}-\left(1-\delta_{j k}\right) \frac{a_{k}}{a_{j}} \frac{2}{\left(r_{k}-r_{j}\right)^{2}},
$$

where $a_{k}$ is given by Eq. (21) and $c_{k}$ is calculated using

$$
c_{k}=a_{k} W\left(r_{k}\right),
$$

where $W(r)=-(2 E+2 Z / r)$ [cf. Eq. (14)]. Finally, combining Eqs. (23)-(28) and using Eq. (20) give us

$$
P_{i j}=\left(1-\delta_{i j}\right) \frac{1}{r_{i}-r_{j}}
$$

and

$$
T_{i j}=-\delta_{i j} \frac{c_{i}}{6 a_{i}}+\left(1-\delta_{i j}\right) \frac{1}{\left(r_{i}-r_{j}\right)^{2}} .
$$

Note that Eq. (22) is satisfied approximately for DVR basis functions constructed from the Coulomb wave function, while Eq. (8) is satisfied exactly for the DVR basis functions constructed from an orthogonal polynomial.

Nevertheless, the CWDVR provides a very accurate discrete spectrum for the $\mathrm{H}$ atom as well as associated physical quantities. ${ }^{32}$ We show later that the CWDVR can also be applied to solve the time-dependent Schrödinger equation very efficiently and accurately. Although the closely related Laguerre DVR or Sturmian basis DVR has been shown by Choi and Light ${ }^{35}$ to give very accurate bound and quasibound states of the $\mathrm{Ar}-\mathrm{HCl}$ complex, this type of DVR gives very poor energies for some bound states of the $\mathrm{H}$ atom [see the discussions in Refs. 18(a), 25, and 32]. In order to describe the time-dependent dynamics of the laser-atom interaction, we have tested different kinds of Laguerre DVRs and found that in many cases they give incorrect ionization rates. The CWDVR is based on a continuum state Coulomb wave function. This may explain its different performance from the Laguerre DVR. We note that Peng et al. ${ }^{13,36}$ did succeed in combining the Laguerre DVR with the FD method to describe a time-dependent laser-molecule interaction using cylindrical coordinates. However, in that work the Laguerre DVR was used to treat the $\rho$ coordinate, which is less important than the $z$ coordinate along which the laser is linearly polarized.

\section{ONE-ELECTRON ATOMIC SYSTEM IN INTENSE LASER FIELDS}

Let us consider an effectively one-electron atomic system in a laser field. In spherical coordinates, the timedependent Schrödinger equation is given by

$$
i \frac{\partial}{\partial t} \Psi(\mathbf{r}, t)=\left[H_{0}(\mathbf{r})+H_{I}(\mathbf{r}, t)\right] \Psi(\mathbf{r}, t),
$$

in which the field-free Hamiltonian $H_{0}$ is defined by

$$
H_{0}=-\frac{1}{2} \nabla^{2}+V_{C}^{l_{0}}(r)=-\frac{1}{2}\left[\frac{1}{r^{2}} \frac{\partial}{\partial r}\left(r^{2} \frac{\partial}{\partial r}\right)-\frac{1}{r^{2}} \hat{L}^{2}\right]+V_{C}^{l_{0}}(r),
$$

where $V_{C}^{l_{0}}(r)$ is the effective Coulomb potential or any kind of short range model potential, which can depend on a fixed value of the angular-momentum quantum number $l_{0}$. [Please note that $V_{C}^{l_{0}}(r)$ is still a spherically symmetric function.] The orbital angular-momentum operator $\hat{L}^{2}$ is defined by

$$
\hat{L}^{2}=-\frac{1}{\sin \theta} \frac{\partial}{\partial \theta}\left(\sin \theta \frac{\partial}{\partial \theta}\right)-\frac{1}{\sin ^{2} \theta} \frac{\partial^{2}}{\partial \phi^{2}},
$$

whose eigenstates are the spherical harmonics $Y_{l m}(\theta, \phi)$.

In Eq. (32), the interaction Hamiltonian $H_{I}(\mathbf{r}, t)$ describes the interaction of the active electron with the applied laser pulse. For the laser parameter ranges of interest in the present work, the dipole approximation is valid. For a laser field linearly polarized along the $z$ axis, $H_{I}(\mathbf{r}, t)$ is given in the length gauge by

$$
H_{I}^{(L)}(\mathbf{r}, t)=\mathbf{d}(r) \cdot \mathbf{E}(t)=d(r) \cos \theta E(t),
$$

with $\mathbf{d}(r)$ being the dipole moment, and in the velocity gauge by

$$
H_{I}^{(V)}(\mathbf{r}, t)=-i \frac{1}{c} \mathbf{A}(t) \cdot \nabla=-i \frac{1}{c} A(t) \nabla_{0},
$$

where $c$ is the speed of light in vacuum. Here $\nabla_{0}$ denotes the zeroth spherical component of the gradient operator $\nabla,{ }^{37}$

$$
\nabla_{0}=\frac{\partial}{\partial z}=\cos \theta \frac{\partial}{\partial r}-\frac{\sin \theta}{r} \frac{\partial}{\partial \theta} .
$$

The electric field strength $\mathbf{E}(t)$ is related to the vector potential $\mathbf{A}(t)$ of the laser pulse by

$$
\mathbf{E}(t)=-\frac{1}{c} \frac{\partial}{\partial t} \mathbf{A}(t) .
$$

\section{A. Discretization of the spatial coordinates}

In order to solve the TDSE, we need to discretize Eq. (32). We expand the angular part of the wave function in terms of spherical harmonics. The radial coordinate $r$ can be discretized in different ways. The most straightforward way is to use the finite difference method, in which case the first and second derivatives with respect to $r$ in Eq. (33) are approximated by formulas involving only several neighboring points. In the present work, however, we expand the radial part of the wave function in the CWDVR basis functions. As 
may be seen from Eqs. (30) and (31), CWDVR is a global method, in which case the representations of the derivatives involve all the grid points. ${ }^{38,39}$

\section{Expansion of the angular part}

Expanding the time-dependent wave function in spherical harmonics,

$$
\Psi(\mathbf{r}, t) \equiv \Psi(r, \theta, \phi, t)=\sum_{l=0}^{L} \sum_{m=-L}^{L} \frac{\varphi_{l m}(r, t)}{r} Y_{l m}(\theta, \phi),
$$

substituting into Eq. (32), multiplying the result by $r Y_{l^{\prime} m^{\prime}}^{*}(\theta, \phi)$, and integrating both sides over angular coordinates, we obtain

$$
\begin{aligned}
i \frac{\partial}{\partial t} \varphi_{l^{\prime} m^{\prime}}(r, t)= & -\frac{1}{2} \frac{d^{2}}{d r^{2}} \varphi_{l^{\prime} m^{\prime}}(r, t)+V_{\mathrm{eff}}^{l}(r) \varphi_{l^{\prime} m^{\prime}}(r, t) \\
& +\left[H_{I}(r, t)\right]_{l^{\prime} m^{\prime}},
\end{aligned}
$$

in which we have defined an effective potential,

$$
V_{\mathrm{eff}}^{l}(r) \equiv V_{C}^{l_{0}}(r)+\frac{l(l+1)}{2 r^{2}},
$$

and the following laser-interaction term,

$$
\begin{aligned}
{\left[H_{I}(r, t)\right]_{l^{\prime} m^{\prime}} \equiv } & \sum_{l=0}^{L} \sum_{m=-L}^{L} \int_{0}^{\pi} \sin \theta d \theta \int_{0}^{2 \pi} d \phi \\
& \times r Y_{l^{\prime} m^{\prime}}^{*}(\theta, \phi) H_{I}(\mathbf{r}, t) \frac{1}{r} \varphi_{l m}(r, t) Y_{l m}(\theta, \phi) .
\end{aligned}
$$

The laser-interaction term in Eq. (42) is gauge dependent. In the length gauge, substituting Eq. (35) into Eq. (42) and making use of the following formula ${ }^{37}$

$$
\cos \theta Y_{l m}(\theta, \phi)=a_{l+1 m} Y_{l+1 m}(\theta, \phi)+a_{l m} Y_{l-1 m}(\theta, \phi),
$$

where

$$
a_{l m}=\sqrt{\frac{(l-m)(l+m)}{(2 l-1)(2 l+1)}},
$$

we arrive at

$$
\begin{aligned}
{\left[H_{I}^{(L)}(r, t)\right]_{l^{\prime} m^{\prime}}=} & d(r) E(t)\left[a_{l^{\prime} m^{\prime}} \varphi_{l^{\prime}-1 m^{\prime}}(r, t)\right. \\
& \left.+a_{l^{\prime}+1 m^{\prime}} \varphi_{l^{\prime}+1 m^{\prime}}(r, t)\right] .
\end{aligned}
$$

In the velocity gauge, substituting Eq. (36) into Eq. (42) and making use of the formula: ${ }^{37}$

$$
\begin{aligned}
\nabla_{0}\left[\frac{1}{r} R(r) Y_{l m}(\theta, \phi)\right]= & a_{l+1 m} Y_{l+1 m}(\theta, \phi) \\
& \times \frac{1}{r}\left(\frac{d}{d r}-\frac{l+1}{r}\right) R(r) \\
& +a_{l m} Y_{l-1 m}(\theta, \phi) \frac{1}{r}\left(\frac{d}{d r}+\frac{l}{r}\right) R(r),
\end{aligned}
$$

we arrive at

$$
\begin{aligned}
{\left[H_{I}^{(V)}(r, t)\right]_{l^{\prime} m^{\prime}}=} & i A(t) \frac{1}{r}\left[l^{\prime} a_{l^{\prime} m^{\prime}} \varphi_{l^{\prime}-1 m^{\prime}}(r, t)\right. \\
& \left.-\left(l^{\prime}+1\right) a_{l^{\prime}+1 m^{\prime}} \varphi_{l^{\prime}+1 m^{\prime}}(r, t)\right] \\
& -i A(t) \frac{d}{d r}\left[a_{l^{\prime} m^{\prime}} \varphi_{l^{\prime}-1 m^{\prime}}(r, t)\right. \\
& \left.+a_{l^{\prime}+1 m^{\prime}} \varphi_{l^{\prime}+1 m^{\prime}}(r, t)\right],
\end{aligned}
$$

with $a_{l^{\prime} m^{\prime}}$ given by Eq. (44).

\section{Discretization of the radial coordinate}

Having changed the TDSE (32) into Eq. (40) (with the interaction term $\left[H_{I}(r, t)\right]_{l^{\prime} m^{\prime}}$ given by Eq. (45) in the length gauge and by Eq. (47) in the velocity gauge), we now address the discretization of the radial coordinate. It is useful for later comparison with the CWDVR method to first review the FD method. In the FD method, the grid points are chosen to be equally spaced,

$$
r_{i}=i \Delta r, \quad i=1,2, \ldots, N .
$$

The five-point central finite difference approximation to the first and second derivatives of $\varphi_{\operatorname{lm}}(r, t)$ are given by ${ }^{13}$

$$
\begin{aligned}
\frac{d}{d r} \varphi_{l m}(r, t)= & \frac{1}{12 \Delta r}\left[\varphi_{l m}(r-2 \Delta r, t)-8 \varphi_{l m}(r-\Delta r, t)\right. \\
& \left.+8 \varphi_{l m}(r+\Delta r, t)-\varphi_{l m}(r+2 \Delta r, t)\right]
\end{aligned}
$$

and

$$
\begin{aligned}
\frac{d^{2}}{d r^{2}} \varphi_{l m}(r, t)= & -\frac{1}{12(\Delta r)^{2}}\left[\varphi_{l m}(r-2 \Delta r, t)\right. \\
& -16 \varphi_{l m}(r-\Delta r, t)+30 \varphi_{l m}(r, t) \\
& \left.-16 \varphi_{l m}(r+\Delta r, t)+\varphi_{l m}(r+2 \Delta r, t)\right] .
\end{aligned}
$$

In order to obtain a better ground state energy, we have used the following approximation for the second derivative at the first grid point in order to properly account for the boundary condition at $r=0:^{40}$

$$
\begin{aligned}
\varphi_{l m}^{\prime \prime}\left(r_{1}, t\right)= & -\frac{1}{12(\Delta r)^{2}}\left[30 \varphi_{l m}\left(r_{1}, t\right)-16 \varphi_{l m}\left(r_{1}+\Delta r, t\right)\right. \\
& \left.+\varphi_{l m}\left(r_{1}+2 \Delta r, t\right)\right]+C_{0} \varphi_{l m}\left(r_{1}, t\right),
\end{aligned}
$$

where $C_{0}$ is a constant that depends on the grid spacing $\Delta r$. For example, taking $C_{0}=-1.48986$ and $\Delta r=0.2$ a.u., we obtain a converged $\mathrm{H}$ ground state energy of -0.500000065 a.u., whereas we must use $C_{0}=-1.814116$ for $\Delta r=0.3$ a.u. in order to get the same value of the ground state energy.

In general, the smaller the spacing, $\Delta r$, the better is the approximation for the derivatives in Eqs. (49) and (50). For atomic systems interacting with intense laser pulses, the electronic wave function can be driven, in general, to hundreds or even thousands of a.u. away from the nucleus. Therefore, one needs a very large number of grid points. Another disadvantage of the FD method is that one needs to deal carefully with the Coulomb singularity at the origin. ${ }^{11,40}$ 
Now let us discretize the coordinate $r$ using the CWDVR basis functions that we discussed in the previous section. As we already mentioned, the CWDVR has several advantages: first, it deals with the singularity of Coulomb-type potentials at $r=0$ naturally and accurately; second, the grid points (i.e., the zeros of the Coulomb wave function) are dense near the origin, where the Coulomb potential plays a crucial role, and sparse at large distances, where it is not very important; third, compared to the FD method, many fewer grid points are needed over the same $r$ range because of the uneven distribution of the grid points.

The CWDVR basis functions $f_{i}(r)$ are given in Eqs. (17), (18), and (22). Let us start from Eq. (40) and expand $\varphi_{l m}(r, t)$ in terms of $f_{i}(r)$ as follows:

$$
\varphi_{l m}(r, t)=\sum_{i=1}^{N} D_{i l m}(t) f_{i}(r),
$$

where the coefficient is given by

$$
\begin{aligned}
D_{i l m}(t) & =\int_{0}^{\infty} d r f_{i}^{*}(r) \varphi_{l m}(r, t) \simeq \sum_{k=1}^{N} \omega_{k} f_{i}^{*}\left(r_{k}\right) \varphi_{l m}\left(r_{k}, t\right) \\
& =\sqrt{\omega_{i}} \varphi_{l m}\left(r_{i}, t\right) .
\end{aligned}
$$

Substituting Eq. (52) into Eq. (40), multiplying both sides by $f_{i^{\prime}}^{*}(r)$, and integrating $r$ over $(0, \infty)$, we obtain

$$
\begin{aligned}
i \frac{\partial}{\partial t} D_{i^{\prime} l^{\prime} m^{\prime}}(t)= & \sum_{i=1}^{N} T_{i^{\prime} i} D_{i l^{\prime} m^{\prime}}(t)+V_{\mathrm{eff}}^{l^{\prime}}\left(r_{i^{\prime}}\right) D_{i^{\prime} l^{\prime} m^{\prime}}(t) \\
& +\left[H_{I}(r, t)\right]_{i^{\prime} l^{\prime} m^{\prime}},
\end{aligned}
$$

where we have made use of Eq. (25). $\left[H_{I}(t)\right]_{i^{\prime} l^{\prime} m^{\prime}}$ stands for the matrix element of the interaction term, which may easily be shown to be given by

$$
\begin{aligned}
{\left[H_{I}^{(L)}(t)\right]_{i^{\prime} l^{\prime} m^{\prime}}=} & \sum_{i=1}^{N} \int_{0}^{\infty} d r f_{i^{\prime}}(r) f_{i}(r) E(t) d(r) \\
& \times\left[a_{l^{\prime} m^{\prime}} D_{i l^{\prime}-1 m^{\prime}}(t)+a_{l^{\prime}+1 m^{\prime}} D_{i l^{\prime}+1 m^{\prime}}(t)\right] \\
= & E(t) d\left(r_{i^{\prime}}\right)\left[a_{l^{\prime} m^{\prime}} D_{i^{\prime} l^{\prime}-1 m^{\prime}}(t)\right. \\
& \left.+a_{l^{\prime}+1 m^{\prime}} D_{i^{\prime} l^{\prime}+1 m^{\prime}}(t)\right],
\end{aligned}
$$

in the length gauge. In the velocity gauge, it takes a slightly more complicated form,

$$
\begin{aligned}
{\left[H_{I}^{(V)}(t)\right]_{i^{\prime} l^{\prime} m^{\prime}}=} & i A(t) \sum_{i=1}^{N} \int_{0}^{\infty} d r f_{i^{\prime}}(r) \frac{1}{r} f_{i}(r)\left[l^{\prime} a_{l^{\prime} m^{\prime}} D_{i l^{\prime}-1 m^{\prime}}(t)-\left(l^{\prime}+1\right) a_{l^{\prime}+1 m^{\prime}} D_{i l^{\prime}+1 m^{\prime}}(t)\right]-i A(t) \\
& \times \sum_{i=1}^{N} \int_{0}^{\infty} d r f_{i^{\prime}}(r) \frac{d}{d r} f_{i}(r)\left[a_{l^{\prime} m^{\prime}} D_{i l^{\prime}-1 m^{\prime}}(t)+a_{l^{\prime}+1 m^{\prime}} D_{i l^{\prime}+1 m^{\prime}}(t)\right] \\
= & i A(t) \frac{1}{r_{i^{\prime}}}\left[l^{\prime} a_{l^{\prime} m^{\prime}} D_{i^{\prime} l^{\prime}-1 m^{\prime}}(t)-\left(l^{\prime}+1\right) a_{l^{\prime}+1 m^{\prime}} D_{i^{\prime} l^{\prime}+1 m^{\prime}}(t)\right]-i A(t) \\
& \times \sum_{i=1}^{N} P_{i^{\prime} i}\left[a_{l^{\prime} m^{\prime}} D_{i l^{\prime}-1 m^{\prime}}(t)+a_{l^{\prime}+1 m^{\prime}} D_{i l^{\prime}+1 m^{\prime}}(t)\right]
\end{aligned}
$$

where we have made use of Eq. (23). The matrix elements of $P_{i^{\prime} i}$ in Eq. (56) and $T_{i^{\prime} i}$ in Eq. (54) are calculated analytically using Eqs. (30) and (31), respectively. The zeros $r_{i}$ needed for evaluating these matrix elements are calculated using COULFG. ${ }^{41}$ Note that for the case of linear laser polarization and the $s$-wave ground state that is considered in this work, the subscript index $m^{\prime}$ is equal to 0 . For this case, then, we have only two dimensional matrices with indices $i^{\prime}$ and $l^{\prime}$.

\section{Distribution of the CWDVR grid points}

The grid point $r_{i}$ of the CWDVR is the solution of $v(r)=0$, where $v(r)$ is defined by $\mathrm{Eq}$. (15) for any given energy $E$ and nuclear charge $Z$. Therefore, the distribution of the CWDVR grid points can be adjusted by the values of the parameters $Z$ and $\kappa$, where $\kappa$ is defined by

$$
\kappa \equiv \sqrt{2 E} .
$$

In Table I, we compare the grid point distributions of the CWDVR for the same maximum grid point value $r_{\max }$ $\simeq 150$ a.u. and for different $\kappa$ values ranging from 0.5 to 5 a.u. The parameter $Z$ is chosen to be either 12 or 20 . We list in this table the number of grid points $N$ (up to the first grid point that is greater than $r=150$ a.u.) and the values of the first and the last grid points, $r_{1}$ and $r_{N}$. We also give three grid spacings, $\Delta r(0), \Delta r(15)$, and $\Delta r(150)$, which cor- 
TABLE I. Comparison of CWDVR grid point distributions for different values of $Z$ and $\kappa$. Note that $\Delta r(r)$ equals the difference of the two grid points closest to $r$.

\begin{tabular}{|c|c|c|c|c|c|c|c|}
\hline & & $\kappa=0.5$ & $\kappa=1.0$ & $\kappa=2.0$ & $\kappa=3.0$ & $\kappa=4.0$ & $\kappa=5.0$ \\
\hline \multirow[t]{6}{*}{$Z=12$} & $N$ & 47 & 64 & 106 & 152 & 198 & 245 \\
\hline & $r_{1}$ & 0.1529 & 0.1526 & 0.1517 & 0.1501 & 0.1480 & 0.1455 \\
\hline & $r_{N}$ & 153.29 & 150.70 & 150.04 & 150.86 & 150.45 & 150.52 \\
\hline & $\Delta r(0)$ & 0.3589 & 0.3565 & 0.3472 & 0.3335 & 0.3171 & 0.2994 \\
\hline & $\Delta r(15)$ & 2.3650 & 1.9090 & 1.3227 & 0.9655 & 0.7491 & 0.6091 \\
\hline & $\Delta r(150)$ & 4.9116 & 2.9158 & 1.5402 & 1.0381 & 0.7815 & 0.6263 \\
\hline \multirow[t]{6}{*}{$Z=20$} & $N$ & 56 & 72 & 112 & 156 & 202 & 248 \\
\hline & $r_{1}$ & 0.09174 & 0.09169 & 0.09148 & 0.09114 & 0.09066 & 0.09007 \\
\hline & $r_{N}$ & 150.27 & 151.39 & 150.74 & 150.47 & 150.73 & 150.43 \\
\hline & $\Delta r(0)$ & 0.2157 & 0.2151 & 0.2130 & 0.2097 & 0.2053 & 0.2001 \\
\hline & $\Delta r(15)$ & 1.8308 & 1.6554 & 1.2137 & 0.9132 & 0.7270 & 0.5972 \\
\hline & $\Delta r(150)$ & 4.3561 & 2.7914 & 1.5209 & 1.0320 & 0.7789 & 0.6250 \\
\hline
\end{tabular}

respond to the spacing between the first two points near 0 , the two points around 15 a.u., and the two nearest grid points in the neighborhood of 150 a.u.

One notices that the value of $Z$ mainly determines the value of the first grid point $r_{1}$, i.e., the greater the $Z$ is, the smaller $r_{1}$ becomes. However, increasing the value of $\kappa$ will mainly decrease the spacing between the grid points at large $r$ and thus one needs more grid points for the same value of $r_{\max }=150$ a.u. At the same time, larger $\kappa$ values result in much more even distributions at larger distances. For example, $\Delta r(15)$ and $\Delta r(150)$ for $\kappa=5$ differ much less than for $\kappa=0.5$.

For the same $r_{\max }=150$ a.u., one observes that the number of the grid points for the CWDVR method is only $1 / 10$ to $1 / 3$ of that for the FD method if we choose $\Delta r=0.2$ a.u. in the FD method (cf. Eq. (48)). As we will discuss below, most of our results for atomic hydrogen are converged for the case of $\kappa=1.0$ and $Z=20$ using only $1 / 10$ the number of grid points required by the FD method $(N=72$ vs $N=750)$.

Some types of nonuniform FD methods, of course, have been designed to solve the time-dependent Schrödinger equation. Comparing the present CWDVR method with these kinds of nonequal-spacing FD methods, the former may not be so superior as it is compared to equal-spacing FD methods. However, nonuniform FD methods may introduce some problems with the Coulomb singularity and with population norm conservation, as discussed by Kono et al. in the conclusions of Ref. 18(b). The present CWDVR is able to overcome such Coulomb singularity problems naturally, and the norm of the population is intrinsically conserved thanks to the fact that the Hamiltonian that is constructed is Hermitian [cf. Eqs. (30), (31) and (54)-(56)].

\section{B. Wave function propagation in time}

After the discretization of the spatial coordinates, one has to advance the initial wave function in discretized time. If the initial wave function is the electronic ground state, it can be computed by the propagation of any trial wave function in imaginary time. The Schrödinger equation in imaginary time becomes a diffusion equation. In this case, any excited state components contained in the trial wave function will decay faster than the ground state. Once the energy is adjusted to the true ground state energy $\varepsilon_{0}$, the asymptotic solution is a steady-state solution. One will thus obtain a converged ground state energy and wave function on the spatial grid after a sufficiently long time of diffusion.

Concerning the propagation of the time-dependent Schrödinger equation, there exist many different methods, such as the split operator, ${ }^{42}$ the Chebyschev polynomial expansion, ${ }^{43}$ the Taylor series, ${ }^{44}$ and the Arnoldi/Lanczos methods. ${ }^{45}$ A number of authors have made detailed comparison studies of the efficiency and accuracy of different propagation schemes. ${ }^{12,13,46}$ Although the choice of the propagation scheme depends on the characteristics of the physical problem at hand, it is generally accepted that for most practical applications the Arnoldi/Lanczos method offers an accurate and flexible approximation of the matrix exponentials involved in the propagation of a wave function. A general introduction to the Arnoldi/Lanczos method can be found in Ref. 47. (Note that whereas the Lanczos method only applies to symmetric or Hermitian matrices, the Arnoldi method applies also to nonsymmetric matrices.)

For the Arnoldi/Lanczos method, an extensive software package, "EXPOKIT," for the computation of a matrix exponential was developed by Sidje, ${ }^{48}$ who provided several alternatives to compute the matrix $e^{t H}$ for small, dense complex matrices $H$. In addition, EXPOKIT has subroutines for computing $e^{t H} \psi_{0}$ for both small, dense matrices and large, sparse matrices. In the present work, we have incorporated this software into our code for the accurate propagation of the wave function for atomic systems interacting with strong laser pulses. The main subroutines we use are "ZGPADM.F" and "ZGEXPV.F." The details of the underlying algorithms and how one uses these subroutines can be found in Ref. 48 .

For all the results presented in this paper, we use the Arnoldi order $M=30$ and the propagation time step $\Delta t$ $=0.01$ a.u. However, we find that for some of the laser parameters we consider, the results are already converged for a much lower order (e.g., $M=12$ ) and much larger time step (e.g., $\Delta t=0.04$ a.u.). 


\section{RESULTS AND DISCUSSIONS}

In this section, we apply the CWDVR method to the ionization of atomic hydrogen by a strong static electric field and by an intense laser field. In order to demonstrate that our method can apply equally to any effectively one-electron atomic system described by a SAE model potential, we also present multiphoton detachment rates for the negative hydrogen ion for several laser frequencies and intensities. In all cases our results are in excellent agreement with other accurate theoretical calculations using different methods.

For an accurate evaluation of the ionization rate for a particular laser intensity, the laser pulse should ramp on adiabatically and remain constant for a sufficiently long time. The laser pulse ramping-on time must be large compared to the initial atomic orbital period of the bound electron so that the electron energy can adjust adiabatically to the rising laser intensity. The constant-intensity time should also be sufficiently long so that the frequency bandwidth is small compared with the laser frequency. Only in this case can one treat the laser field as monochromatic. We assume the vector potential $\mathbf{A}(t)$ of a linearly polarized laser to have the following form:

$$
\mathbf{A}(t)=A_{0} f(t) \cos (\omega t) \hat{\mathbf{k}},
$$

where the polarization vector $\hat{\mathbf{k}}$ is along the $z$ axis and the pulse envelope, $f(t)$, is given by

$$
f(t)=\left\{\begin{array}{l}
\frac{1}{2}\left[1-\cos \left(\frac{\pi t}{\tau_{1}}\right)\right], \quad 0 \leqslant t \leqslant \tau_{1} \\
1, \quad \tau_{1} \leqslant t \leqslant \tau_{1}+\tau_{2},
\end{array}\right.
$$

where $\tau_{1}$ and $\tau_{2}$ are taken to be 5-10 and 10-40 laser cycles, respectively, depending on the laser frequency under consideration. The peak value of the vector potential $A_{0}$ is connected to the peak laser intensity $I_{0}$ by

$$
A_{0}=\frac{E_{0}}{\omega}=\frac{1}{\omega} \sqrt{\frac{I_{0}}{I_{\mathrm{au}}}},
$$

where $E_{0}$ is the maximum electric field strength of the laser field and the atomic unit of laser intensity $I_{\text {au }}$ equals $3.5094 \times 10^{16} \mathrm{~W} / \mathrm{cm}^{2}$. The electric field strength $\mathbf{E}(t)$ is calculated using Eq. (38).

In order to avoid reflection of the wave function at the outer boundary of the $r$ grid, the wave function is multiplied by an absorbing function at each propagation step, as follows:

$$
\Psi_{\text {abs }}(\mathbf{r}, t)=M(r) \Psi(\mathbf{r}, t),
$$

in which $\Psi_{\text {abs }}$ is the wave function after the application of the absorption function $M(r)$ given by

$$
M(r)=\left\{\begin{array}{cc}
1, & r \leqslant r_{\alpha} \\
\exp \left[-\left(\frac{r-r_{\alpha}}{r_{\sigma}}\right)^{2}\right], & r>r_{\alpha},
\end{array}\right.
$$

where $r_{\alpha}=\alpha r_{\max }$ and $r_{\sigma}=\sigma r_{\max }$ with $r_{\max }$ being the maximum value of the grid. It is very important to carefully choose the absorbing parameters $\alpha$ and $\sigma$ such that the function $M(r)$ is sufficiently smooth and that the wave function near the edge is completely absorbed without any reflection in order to avoid any unphysical effects induced by the absorbing potential. ${ }^{49}$ Based on our experience, we take $0.3 \leqslant \alpha \leqslant 0.6$ and $0.5 \leqslant \sigma \leqslant 5.0$ depending on the value of $r_{\max }$ and the laser parameters. Our criterion is that the time-dependent physical quantities, such as the population decay of the ground state, should be converged against any small variations of these parameters about their chosen values. Of course, convergence must be achieved also with respect to variations of other parameters, such as the maximum value of the angular momentum $L$, the number of grid points $N$ in the $r$ coordinate, the propagation time step $\Delta t$, the Arnoldi propagator order $M$, etc.

\section{A. Choice of gauge}

Although the use of alternative gauges describing the interaction Hamiltonian $H_{I}(t)$ should, in principle, lead to the same physical results, it is not true in practice because of the use of approximations and because of inaccuracies in the numerical wave functions. For some approximate methods, the length gauge proves to be superior to the velocity gauge in some circumstances. ${ }^{50}$ However, as discussed by Cormier and Lambropoulos, ${ }^{51}$ the velocity gauge is preferable for some other numerical methods owing to the fact that the canonical momentum in the velocity gauge can be reduced to a slowly varying variable. In this case, one avoids having widely varying variables. Especially in our present case in which the wave function is expanded in terms of spherical harmonics, we find that a much smaller maximum value of $L$ is needed for a converged result in the velocity gauge than that needed in the length gauge. ${ }^{11}$

In the present work, we have employed the velocity gauge for the case of atomic hydrogen in a laser field. However, we have employed the length gauge to treat the cases of atomic hydrogen in a static electric field and the $\mathrm{H}^{-}$ion in a laser field. One of the reasons for the latter choice is that it is easier for us to compare our results with previous calculations that also use the length gauge. Another reason, for $\mathrm{H}^{-}$, is that the angular-momentum-dependent model potential ${ }^{52}$ makes it impossible to obtain a simple expression for the potential in the velocity gauge. ${ }^{53}$

\section{B. Calculation of physical observables}

In principle, once the time-dependent wave function is computed at each time step, one can easily calculate any physical observable. For instance, the population remaining in the whole box, $P_{\text {tot }}(t)$, is given by

$$
\begin{aligned}
P_{\mathrm{tot}}(t) & =\int_{0}^{\infty} r^{2} d r \int_{0}^{\pi} \sin \theta d \theta \int_{0}^{2 \pi} d \phi|\Psi(r, \theta, \phi, t)|^{2} \\
& =\sum_{i l m}\left|D_{i l m}(t)\right|^{2},
\end{aligned}
$$

where we have made use of Eqs. (39) and (52). The population remaining in a sphere of a certain radius is given simply by summing over those $r$ grid points within the sphere. 
Knowing the time-dependent population, $P(t)$, one can estimate the ionization rate $\Gamma$ by assuming that the population decays exponentially,

$$
P(t)=P\left(t_{0}\right) \exp \left[-\left(t-t_{0}\right) \Gamma\right],
$$

which implies that

$$
\Gamma=-\frac{1}{t-t_{0}} \log \left[P(t) / P\left(t_{0}\right)\right],
$$

where $t_{0}$ is usually the starting time of the flat part of the laser pulse.

\section{Convergence of the CWDVR method}

Since the CWDVR grid depends on the two parameters $Z$ and $\kappa$, one must make sure that any calculation gives converged results with respect to variations of these parameters. As shown in Table I, the CWDVR grid for smaller $\kappa$ is much coarser. At the same time, the first point of the grid is mainly decided by the value of $Z$. It is expected that a much finer CWDVR grid will give a better representation of the Coulomb potential and of the laser-driven electronic wave function.

In order to illustrate the convergence of the CWDVR wave function, multiphoton ionization of $\mathrm{H}$ by an infrared laser having a moderate intensity serves as a good example. For a hydrogenic atom, the potential is given by

$$
V_{C}^{l_{0}}(r)=-\frac{Z_{n}}{r}
$$

where $Z_{n}$ is the nuclear charge, which equals unity for the $\mathrm{H}$ atom. The dipole moment in this case is given by $\mathbf{d}(r)=\mathbf{r}$. For all CWDVR grids considered in Table I, we obtain an $\mathrm{H}$ atom ground state energy of -0.49999997 a.u. or better.

In Fig. 1, we present the natural logarithm of the population within a sphere of radius $r=25$ a.u. calculated by the FD method and by the CWDVR method corresponding to different values of $Z$ and $\kappa$. We consider a laser pulse having a wavelength $\lambda=780 \mathrm{~nm}$, a peak intensity of $I_{0}=2$ $\times 10^{14} \mathrm{~W} / \mathrm{cm}^{2}$, a five-cycle ramp-up time, and a ten-cycle flat top. The maximum value of the angular momentum $L$ is taken to be 20 in order to obtain a converged result. In the FD case, the grid spacing $\Delta r$ is taken to be 0.2 a.u., and the number of grid points $N=750$ for the maximum value of the grid point, $r_{\max }=150$ a.u. The corresponding numbers for the $r$ grid points used for the CWDVR cases are indicated in Table I. From Fig. 1, we conclude that our results are indeed converged to the FD difference results as $\kappa$ is increased from 0.5 to 2.0. Even for the coarsest case, $\kappa=0.5$, where only 56 grid points are used, the result is reasonably good. The ionization rate estimated from this latter curve is 4.63 $\times 10^{13} \mathrm{~s}^{-1}$, which is close to the FD result of 5.07 $\times 10^{13} \mathrm{~s}^{-1}$; for $\kappa=2$ and $Z=20$ the CWDVR result is 5.05 $\times 10^{13} \mathrm{~s}^{-1}$. We also observe that, for the present case, the results are not very sensitive to variations of the value of $Z$ (for the same value of $\kappa$ ). However, we find that, in most cases considered later, $Z=20$ is usually preferable for its better representation of the Coulomb potential near $r=0$.

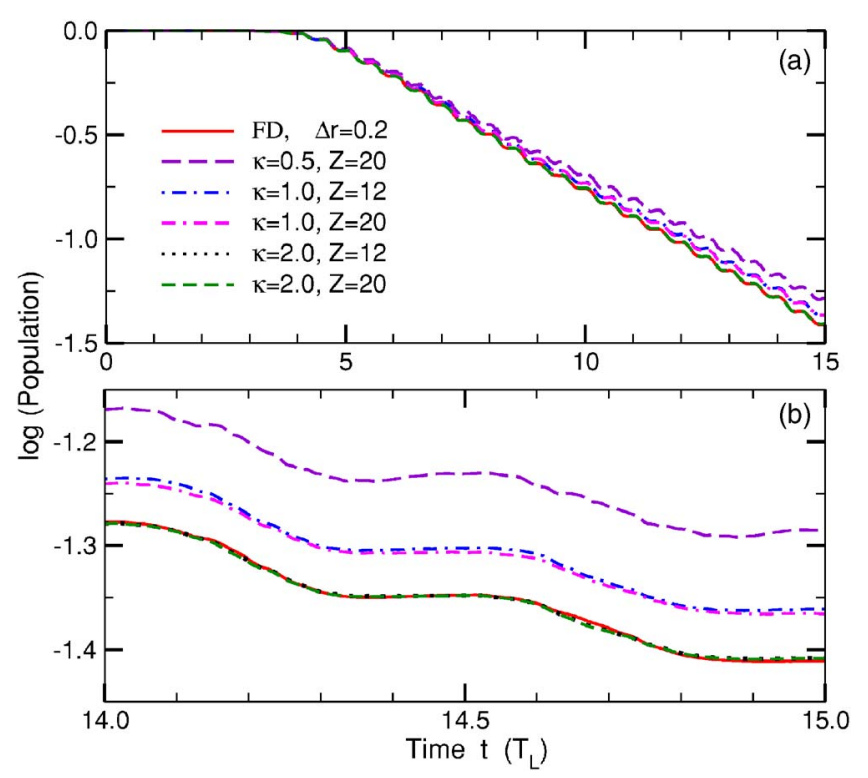

FIG. 1. (Color online) Convergence of the CWDVR method for different $\kappa$ and $Z$ values. The natural logarithm of the population as a function of time (in units of the laser period $T_{L}=2 \pi / \omega$ ) is shown for a laser wavelength of $780 \mathrm{~nm}$ and a peak laser intensity of $2 \times 10^{14} \mathrm{~W} / \mathrm{cm}^{2}$. (a) Results for different CWDVR grids (with $\kappa$ and $Z$ as indicated) are compared to the result of the FD method for time $t$ in the range $0 \leqslant t / T_{L} \leqslant 15$. (b) A magnified version of (a) for time $t$ in the range $14 \leqslant t / T_{L} \leqslant 15$. Note that in both panels the three curves representing the FD result and the two $\kappa=2$ CWDVR results are nearly indistinguishable on the scale of the figure and appear as the lowest curve in each panel.

In practice, the ionization rate is fitted by an exponential decay of the ground state population or the decay of the total population within some sphere of radius $r$ in the constant value region of the laser pulse. In the present calculations, we have defined an inner sphere and a middle sphere with radii $r_{a}=25$ and $r_{b}=50$ a.u., respectively. The population remaining within these two spheres, $P_{a}$ and $P_{b}$, respectively, together with the population in the ground state, $P_{g}$, and the total population in the entire box with $r \approx 150$ a.u., $P_{\text {tot }}$, are recorded in each time time step. As an example, we show in Fig. 2 results for the ionization of $\mathrm{H}$ by a laser of frequency of 0.6 a.u. and peak intensity of $4.375 \times 10^{13} \mathrm{~W} / \mathrm{cm}^{2}$. The laser ramps on over 5 cycles and remains constant for 50 cycles. We observe that the populations within different spheres of $r$ exhibit exponential decays with time given by straight lines parallel to the line for ground state decay. It does not therefore matter in this case which curve is used to estimate the ionization rate. Our calculated ionization rate is $1.5658 \times 10^{-3}$ a.u., which is in very good agreement with Chu and Cooper's result of $1.5672 \times 10^{-3}$ a.u. (Ref. 54).

In a similar way, we have also estimated the multiphoton ionization rate of $\mathrm{H}$ by a laser of wavelength of $1064 \mathrm{~nm}$ and peak intensity of $1 \times 10^{14} \mathrm{~W} / \mathrm{cm}^{2}$. The result is 2.97 $\times 10^{12} \mathrm{~s}^{-1}$, which is in good agreement with an independent molecular code result of $2.85 \times 10^{12} \mathrm{~s}^{-1}$ (Ref. 36) in cylindrical coordinates and the FD result of $2.92 \times 10^{12} \mathrm{~s}^{-1}$ in spherical coordinates. ${ }^{36}$

\section{Multiphoton ionization of $\mathbf{H}$ by intense laser pulses}

In this section, we provide more rigorous tests of the present method by calculating the ionization rates of $\mathrm{H}$ for a 


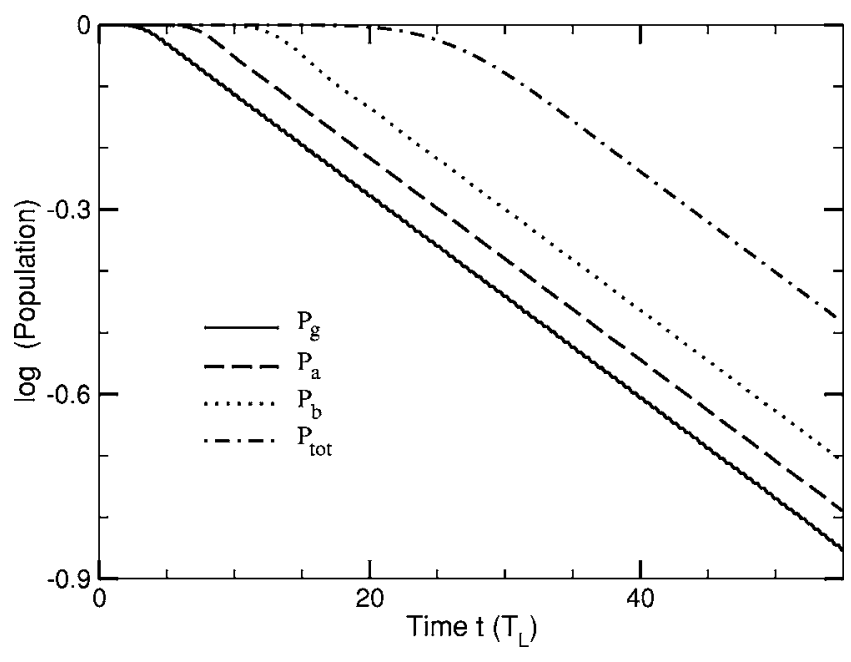

FIG. 2. The natural logarithm of the decay of the ground state and of populations within different spheres of radius $r$ for ionization of atomic $\mathrm{H}$ by a laser having frequency $\omega=0.6$ a.u., peak intensity $I_{0}=4.375$ $\times 10^{13} \mathrm{~W} / \mathrm{cm}^{2}$, a 5 -cycle ramp up, and a 50 -cycle flat top $\left(T_{L}=2 \pi / \omega\right) . P_{g}$ $=$ population remaining in the ground state, $P_{a}\left(P_{b}\right)=$ population remaining within a sphere of radius $r_{a}=25$ a.u. $\left(r_{b}=50\right.$ a.u. $)$, and $P_{\text {tot }}=$ population remaining within the entire grid, $r \approx 150$ a.u.

large number of wavelengths from very low to very high peak intensities. For all the calculations presented here, we use the CWDVR grid corresponding to $\kappa=1.0$ and $Z=20$. The number of grid points is $N=72$ and $r_{\max }=151.39$ a.u. The maximum angular momentum, $L=10$, gives converged results in all cases when the velocity gauge is employed for the interaction Hamiltonian. The parameters $\alpha$ and $\sigma$ for the absorbing function in Eq. (62) are taken to be $\alpha=0.4$ and $\sigma=4$. In Table II, we compare ionization rates calculated using the present method with other theoretical results. On the whole our results agree best with those of Chu and Cooper, ${ }^{54}$ who employed an ab initio Floquet method. However, the present time-dependent results are also in reasonable agreement with those of the TDSE calculations of Ref. 55 using a complex Sturmian basis and those of Ref. 10 using a FD method. The results for $\omega=0.2$ a.u. for intensities of $1.75 \times 10^{14}$ and $3.94 \times 10^{14} \mathrm{~W} / \mathrm{cm}^{2}$ are in less favorable agreement, because the high ionization rates lead to very fast decay of the ground population and thus estimates of any TDSE method become less accurate.
TABLE II. Ionization rate $\Gamma$ for ionization of $\mathrm{H}$ by a linearly polarized laser of intensity $I_{0}$ and frequency $\omega$. The present results are compared with the results of Chu and Cooper, (Ref. 54), Pont et al. (Ref. 55), and Kulander (Ref. 10). Intensities and ionization rates are presented in the form $p(q)$ $\equiv p \times 10^{q}$.

\begin{tabular}{cccccc}
\hline \hline & & \multicolumn{4}{c}{$\Gamma\left(\mathrm{s}^{-1}\right)$} \\
\cline { 3 - 6 } $\begin{array}{c}\omega \\
(\text { a.u. })\end{array}$ & $\begin{array}{c}I_{0} \\
\left(\mathrm{~W} / \mathrm{cm}^{2}\right)\end{array}$ & Present & Ref. 54 & Ref. 55 & Ref. 10 \\
\hline \multirow{2}{*}{0.55} & $7.00(12)$ & $1.43(13)$ & $1.43(13)$ & $1.43(13)$ & $1.4(13)$ \\
0.28 & $7.00(12)$ & $3.73(11)$ & $3.73(11)$ & $4.0(11)$ & $3.3(11)$ \\
& $4.38(13)$ & $1.33(13)$ & $1.33(13)$ & $1.35(13)$ & $1.2(13)$ \\
0.20 & $4.38(13)$ & $3.74(12)$ & $3.86(12)$ & $4.0(12)$ & $2.8(12)$ \\
& $1.75(14)$ & $2.65(14)$ & $2.89(14)$ & $2.7(14)$ & $4.0(14)$ \\
& $3.94(14)$ & $6.14(14)$ & $5.65(14)$ & $6.0(14)$ & $7.0(14)$ \\
\hline \hline
\end{tabular}

In Table III, we compare our results with the benchmark calculations of Chu and Cooper $^{54}$ using the ab initio nonperturbative $L^{2}$ non-Hermitian Floquet method. The laser frequency $\omega$ varies from 0.6 to 0.26 a.u., which corresponds to one-to two-photon ionization of the ground state of atomic H. Note that $E_{0}$ is related to $F_{\text {rms }}$ as follows:

$$
E_{0}=\sqrt{2} F_{\mathrm{rms}}=\sqrt{I_{0} / I_{\mathrm{au}}} \text {. }
$$

As may be seen, there is very good agreement between the present CWDVR results and those of Ref. 54 over these wide ranges of laser parameters.

\section{E. Ionization of $\mathbf{H}$ by static electric fields}

Now we turn to ionization of $\mathrm{H}$ (in its ground state) by an abruptly turned-on static electric field. The excitation and ionization dynamics of atoms and molecules by static electric fields has been of great importance since the foundation of quantum mechanics ${ }^{56}$ and continues to be of great current interest. ${ }^{57-59}$ Although the long time behavior is dominated by the exponential decay of the ground state, large deviations from exponential decay are expected owing to the sudden turn on of the static electric field. The Hamiltonian of this system is unbounded from below. There are also many resonances present. Deviations from exponential decay are expected to be large in the case of strong static fields. ${ }^{59}$ How-

TABLE III. Multiphoton ionization rates for $\mathrm{H}$ for four different laser electric field strengths, $F_{\mathrm{rms}}=E_{0} / \sqrt{2}$, and seven photon energies $\omega$. Present results are compared with those of Chu and Cooper (Ref. 54) (who used a nonperturbative $L^{2}$ non-Hermitian Floquet method).

$\Gamma / 2$ (a.u.)

\begin{tabular}{|c|c|c|c|c|c|c|c|c|}
\hline \multirow{2}{*}{$\begin{array}{c}\omega \\
\text { (a.u.) }\end{array}$} & \multicolumn{2}{|c|}{$F_{\mathrm{rms}}=0.01$ a.u. } & \multicolumn{2}{|c|}{$F_{\mathrm{rms}}=0.025$ a.u. } & \multicolumn{2}{|c|}{$F_{\text {rms }}=0.05$ a.u. } & \multicolumn{2}{|c|}{$F_{\mathrm{rms}}=0.075$ a.u. } \\
\hline & Present & Ref 54 & Present & Ref 54 & Present & Ref 54 & Present & Ref 54 \\
\hline 0.60 & $0.125(-3)$ & $0.125(-3)$ & $0.783(-3)$ & $0.784(-3)$ & $0.313(-2)$ & $0.314(-2)$ & $0.704(-2)$ & $0.711(-2)$ \\
\hline 0.55 & $0.173(-3)$ & $0.173(-3)$ & $0.108(-2)$ & $0.108(-2)$ & $0.435(-2)$ & $0.436(-2)$ & $0.982(-2)$ & $0.989(-2)$ \\
\hline 0.50 & $0.250(-3)$ & $0.247(-3)$ & $0.157(-2)$ & $0.154(-2)$ & $0.647(-2)$ & $0.624(-2)$ & $0.149(-1)$ & $0.139(-1)$ \\
\hline 0.30 & $0.378(-5)$ & $0.377(-5)$ & $0.131(-3)$ & $0.131(-3)$ & $0.160(-2)$ & $0.161(-2)$ & $0.594(-2)$ & $0.639(-2)$ \\
\hline 0.28 & $0.451(-5)$ & $0.451(-5)$ & $0.161(-3)$ & $0.161(-3)$ & $0.204(-2)$ & $0.204(-2)$ & $0.821(-2)$ & $0.815(-2)$ \\
\hline 0.27 & $0.503(-5)$ & $0.502(-5)$ & $0.180(-3)$ & $0.180(-3)$ & $0.230(-2)$ & $0.231(-2)$ & $0.920(-2)$ & $0.920(-2)$ \\
\hline 0.26 & $0.562(-5)$ & $0.562(-5)$ & $0.202(-3)$ & $0.202(-3)$ & $0.256(-2)$ & $0.261(-2)$ & $0.106(-1)$ & $0.110(-1)$ \\
\hline
\end{tabular}




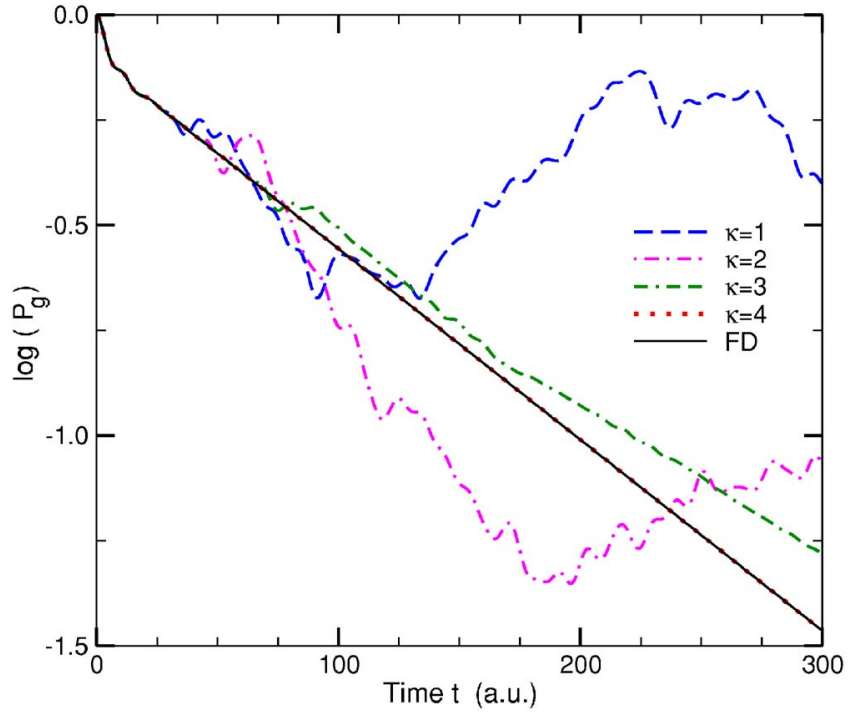

FIG. 3. (Color online) Depletion of the ground state of $\mathrm{H}$ by a static electric field having field strength of 0.08 a.u. The natural logarithm of the ground state probability $P_{g}$ is shown as a function of the field duration. Results calculated by different CWDVR grids for different $\kappa$ values $(Z=20$ in each case) are compared against the result calculated by the FD method with $\Delta r=0.1$ a.u. Note that the $\kappa=4$ result is indistinguishable from the FD result on the scale of this figure.

ever, there is a substantially long transition time region for weak and intermediate fields as well, whose length depends on the particular field strength.

In order to investigate this transition regime, one has to make sure that the nonexponential decay indeed comes from physical dynamics rather than any numerical antifacts such as nonconvergence of the results due to the choice of the grid or reflection of the wave function from the edge of the grid. These matters are extremely important for the static electric field case because the wave packet is driven away in one direction rather than in an oscillatory fashion, as in the case of a laser pulse. In Fig. 3, we show the natural logarithm of the ground state population of $\mathrm{H}$ in the presence of a static electric field $F=0.08$ a.u. for different CWDVR grids and for the FD method. Note that we use the length gauge in this case for the dipole interaction. The maximum of the grid, $r_{\max }$, for all cases is taken to be around 150 a.u. and the number of grid points in each case is listed in Table I. We take $Z=20$ for every CWDVR grid. In addition, the absorbing function parameters, $\alpha$ and $\sigma$, are taken to have the extreme values of 0.25 and 0.4 , respectively, in order to avoid reflection from the edge. For this field strength, we obtain converged results only if $\kappa \geqslant 4.0$, in which case the grid is dense enough to provide a good representation of the interaction term using the length gauge. Note that the performance of the present CWDVR is much better than the DVR method used by Dimitrovski et al., ${ }^{60}$ who obtain converged results only for $t \leqslant 40$ a.u. Therefore, one has to be very careful not to interpret the nonexponential decay for $\kappa \leqslant 3.0$ as originating from any physical excitation or ionization dynamics. $^{58,59}$

Using the CWDVR method and parameters $\kappa \geqslant 4.0$ and $Z=20$, we have studied short-time dynamics for other field strengths. In Fig. 4, we present the population decay of the ground state for different electric field strengths $F$. For the lowest field strength, $F=0.005$ a.u., one observes oscillations on both small- and large-time scales. For the case when $F$ becomes eight times that in Fig. 4(a) [cf. Fig. 4(b)], we observe a quadratic decay in time ${ }^{59}$ for $0 \leqslant t \leqslant 8$ a.u., followed by an irregular transition region before the system becomes stable for $t \geqslant 90$ a.u. As the field strength increases further in Figs. 4(c) and 4(d), the transition region becomes shorter and shorter and is followed by purely exponential decay. The transition regions are shown by Durand and Paidarova ${ }^{59}$ to be directly related to $2 s-2 p$ resonances by inspecting the spectral density line shape. Note that the fast quadratic decay in time ${ }^{59}$ is present for (c) and (d) as well when the field is turned on.

It is remarkable that the present time-dependent calculations for the entire region from very weak to very strong field strengths are in complete agreement with the results obtained using complex scaling methods. ${ }^{57,59}$ This is further confirmed by comparing our ionization rates fitted by an expo-

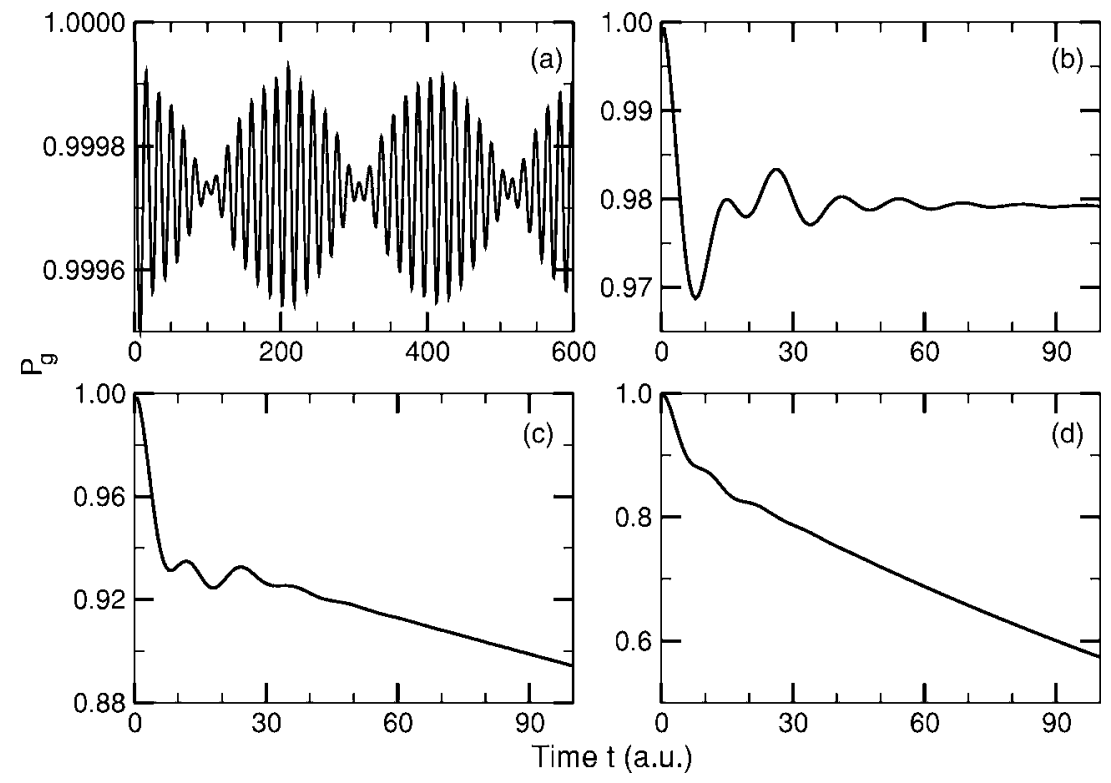

FIG. 4. $\mathrm{H}$ ground state survival probability, $P_{g}$, as a function of time in a static electric field. The field strength $F$ is taken to be (a) 0.005 , (b) 0.04 , (c) 0.06 , and (d) 0.08 a.u. These results are in perfect agreement with those by Durand and Paidarova (Ref. 59) and by Scrinzi (Ref. 57) (neither of which are shown here because they are indistinguishable from ours on the scale of the figures). 
TABLE IV. Ionization rate $\Gamma$ (in a.u.) for ionization of the ground state of $\mathrm{H}$ by a static electric field of strength $F$. Results are compared with those of Scrinzi (Ref. 57), Peng et al. (Ref. 13), and Bauer and Mulser (Ref. 61).

\begin{tabular}{lllll}
\hline \hline & \multicolumn{5}{c}{$F$ (a.u.) } \\
\cline { 2 - 5 } Calculations & 0.06 & 0.08 & 0.1 & 0.5 \\
\hline Present & $5.1509(-4)$ & $4.5396(-3)$ & $1.42(-2)$ & $5.64(-1)$ \\
Ref. 57 & $5.1508(-4)$ & $4.5397(-3)$ & $1.45(-2)$ & $5.60(-1)$ \\
Ref. 13 & $5.15(-4)$ & $4.55(-3)$ & $\cdots$ & $\cdots$ \\
Ref. 61 & $\cdots$ & $\cdots$ & $1.2(-2)$ & $5.4(-1)$ \\
\hline \hline
\end{tabular}

nential decay in the region following the transition region, as shown in Table IV. Also shown in this table are the results of some other time-dependent calculations. ${ }^{13,61}$

\section{F. Multiphoton detachment rates for $\mathrm{H}^{-}$by a strong laser pulse}

In order to show that the present method can apply equally well to any atomic system described by an appropriate SAE model potential, we study in this section multiphoton detachment of the negative ion of hydrogen. For $\mathrm{H}^{-}$, we use the angular-momentum-dependent model potential proposed by Laughlin and $\mathrm{Chu},{ }^{52}$

$$
V_{C}^{l_{0}}(r)=\left(1+\frac{1}{r}\right) e^{-2 r}-\frac{\alpha_{d}}{2 r^{4}} W_{6}\left(r / r_{c}\right)+u_{l_{0}}(r),
$$

where

$$
\begin{aligned}
& W_{j}(x)=1-e^{-x^{j}}, \\
& u_{l_{0}}(r)=\left(c_{0}+c_{1} r+c_{2} r^{2}\right) e^{-\beta r} .
\end{aligned}
$$

In the present calculations, we use the same parameter values (which depend on the value of $l_{0}$ ) as listed in Table I of Ref. 52. The length gauge is used to describe the interaction term. Note also that we employ a dipole operator that accounts for polarization of the hydrogen-atom core by the outer, loosely bound electron, ${ }^{52}$ i.e., $\mathbf{d}(r)$ in this case is given by

$$
\mathbf{d}(r)=\left[1-\frac{\alpha_{d}}{r^{3}} W_{3}\left(r / r_{c}\right)\right] \mathbf{r} \text {. }
$$

Using this model potential, we obtain a ground state energy of -0.027730 a.u. for all CWDVR grids listed in Table I; this is in very good agreement with the value of -0.027733 a.u. calculated by Telnov and $\mathrm{Chu}^{62}$ and with the experimentally measured value of -0.027716 a.u.. (Ref. 63).

In Table $\mathrm{V}$, we compare the total detachment rates of $\mathrm{H}^{-}$ calculated using the present method with results of other theoretical calculations. We obtain converged results for CWDVR grid parameters $\kappa=2$ and $Z=20$. It is not surprising that the required grid is less demanding for the $\mathrm{H}^{-}$detachment case than for the $\mathrm{H}$ atom in a static electric field case, since in the former case we have a short range potential. For all laser parameters considered in Table $\mathrm{V}$, we obtain very good agreement with results of Refs. 64-66.

Note that, instead of Eq. (71), Telnov and Chu use the dipole moment $\mathbf{d}(r)=\mathbf{r}$, which may account for the slight differences between their results and ours. We have calculated some results by our CWDVR method using the latter dipole moment. For example, we obtain a detachment rate of $4.51 \times 10^{-4}$ a.u. instead of $4.43 \times 10^{-4}$ a.u. for $1064 \mathrm{~nm}$ at $1 \times 10^{11} \mathrm{~W} / \mathrm{cm}^{2}$, which is then much closer to the corresponding result of Ref. 65. On the whole, however, our results are slightly closer to those of Haritos et al. ${ }^{64}$ which are calculated by solving the time-independent Schrödinger equation by the nonperturbative many-electron, manyphoton theory (MEMPT). This better agreement may be due to the fact that the dipole moment we use takes the core polarization effects into account.

\section{CONCLUSIONS}

We have presented an accurate and efficient method for solving the time-dependent Schrödinger equation for the case of an effective one-electron atomic system described by an appropriate model potential. Compared to the usual FD dis-

TABLE V. Multiphoton detachment rates for $\mathrm{H}^{-}$for laser wavelengths $\lambda=1064,1640$, and $1908 \mathrm{~nm}$ and 11 intensities (ranging from $1 \times 10^{10}$ to 1

\begin{tabular}{|c|c|c|c|c|c|c|c|c|c|}
\hline \multirow{2}{*}{$\begin{array}{l}\text { Intensity } \\
\left(\mathrm{W} / \mathrm{cm}^{2}\right)\end{array}$} & \multicolumn{3}{|c|}{$1064 \mathrm{~nm}$} & \multicolumn{3}{|c|}{$1640 \mathrm{~nm}$} & \multicolumn{3}{|c|}{$1908 \mathrm{~nm}$} \\
\hline & Present & Ref 64 & Ref 65 & Present & Ref 64 & Ref 66 & Present & Ref 64 & Ref 66 \\
\hline $1 \times 10^{10}$ & $4.56(-5)$ & $4.50(-5)$ & $4.65(-5)$ & $6.5(-7)$ & $5.05(-7)$ & $2.98(-7)$ & $4.90(-7)$ & $4.33(-7)$ & $4.80(-7)$ \\
\hline $5 \times 10^{10}$ & $2.25(-4)$ & $2.20(-4)$ & $\cdots$ & $7.7(-6)$ & $6.82(-6)$ & $\cdots$ & $1.14(-5)$ & $1.04(-5)$ & $\cdots$ \\
\hline $8 \times 10^{10}$ & $3.57(-4)$ & $3.56(-4)$ & $\cdots$ & $1.81(-5)$ & $1.71(-5)$ & $\cdots$ & $2.81(-5)$ & $2.58(-5)$ & $\cdots$ \\
\hline $1 \times 10^{11}$ & $4.43(-4)$ & $4.43(-4)$ & $4.53(-4)$ & $2.79(-5)$ & $2.63(-5)$ & $2.78(-5)$ & $4.27(-5)$ & $3.98(-5)$ & $4.37(-5)$ \\
\hline $2 \times 10^{11}$ & $8.58(-4)$ & $8.70(-4)$ & $\ldots$ & $1.01(-4)$ & $0.99(-4)$ & $1.05(-4)$ & $1.55(-4)$ & $1.46(-4)$ & $1.58(-4)$ \\
\hline $3 \times 10^{11}$ & $1.25(-3)$ & $1.28(-3)$ & $\ldots$ & $2.14(-4)$ & $2.10(-4)$ & $\cdots$ & $3.23(-4)$ & $3.01(-4)$ & $\cdots$ \\
\hline $4 \times 10^{11}$ & $1.61(-3)$ & $1.66(-3)$ & $\cdots$ & $3.68(-4)$ & $3.55(-4)$ & $3.76(-4)$ & $5.18(-4)$ & $4.95(-4)$ & $5.30(-4)$ \\
\hline $6 \times 10^{11}$ & $2.24(-3)$ & $2.38(-3)$ & $\cdots$ & $7.62(-4)$ & $7.19(-4)$ & $\cdots$ & $9.52(-4)$ & $9.78(-4)$ & $\cdots$ \\
\hline $8 \times 10^{11}$ & $2.66(-3)$ & $2.98(-3)$ & $\cdots$ & $1.16(-3)$ & $1.15(-3)$ & $1.23(-3)$ & $1.48(-3)$ & $1.55(-3)$ & $1.52(-3)$ \\
\hline $9 \times 10^{11}$ & $2.81(-3)$ & $3.24(-3)$ & $\cdots$ & $1.39(-3)$ & $1.40(-3)$ & $\cdots$ & $1.72(-3)$ & $1.88(-3)$ & $\ldots$ \\
\hline $1 \times 10^{12}$ & $3.05(-3)$ & $3.46(-3)$ & $2.95(-3)$ & $1.64(-3)$ & $1.64(-3)$ & $1.73(-3)$ & $2.16(-3)$ & $2.12(-3)$ & $2.18(-3)$ \\
\hline
\end{tabular}
$\times 10^{12} \mathrm{~W} / \mathrm{cm}^{2}$ ). The present results using the CWDVR method are compared with results of Haritos et al. (Ref. 64) and of Telnov and Chu. (Refs. 65 and 66 ). The detachment rates are given in the form of $p(q) \equiv p \times 10^{q}$.

Photodetachment Rate $\Gamma$ (a.u.) 
cretization method of the radial coordinate, the present CWDVR method requires three to ten times fewer grid points and treats the Coulomb singularity naturally and accurately. As examples, our method has been shown to provide accurate multiphoton ionization or detachment rates for both $\mathrm{H}$ and $\mathrm{H}^{-}$for a variety of laser field parameters, as evidenced by very good agreement with results of other accurate theoretical calculations. We have also applied our method to investigate the short-time excitation and ionization dynamics of $\mathrm{H}$ in both weak and strong static electric fields. The ground state survival probabilities and the ionization rates calculated using the present method are in excellent agreement with results obtained using complex rotation methods. Since the CWDVR method treats the Coulomb potential accurately and needs fewer points in the radial coordinate $r$, the present method may open the way to more efficient treatments for time-dependent processes in manyelectron atomic systems.

\section{ACKNOWLEDGMENTS}

One of the authors (L.-Y.P.) gratefully acknowledges helpful discussions with D. A. Telnov and S. I. Chu concerning the choice of gauge for multiphoton detachment of $\mathrm{H}^{-}$ and with R. B. Sidje concerning use of the package "EXPOKIT." This work was supported in part by the U.S. Department of Energy, Office of Science, Division of Chemical Sciences, Geosciences, and Biosciences, under Grant No. DE-FG03-96ER14646.

${ }^{1}$ T. Brabec and F. Krausz, Rev. Mod. Phys. 72, 545 (2000).

${ }^{2}$ M. Protopapas, C. H. Keitel, and P. L. Knight, Rep. Prog. Phys. 60, 389 (1997).

${ }^{3}$ P. Agostini and L. F. DiMauro, Rep. Prog. Phys. 67, 813 (2004); A. Scrinzi, M. Yu. Ivanov, R. Kienberger, and D. M. Villeneuve, J. Phys. B 39, R1 (2006).

${ }^{4}$ A. Föhlisch, P. Feulner, F. Hennies, A. Fink, D. Menzel, D. SanchezPortal, P. M. Echenique, and W. Wurth, Nature (London) 436, 373 (2005); M. Wickenhauser, J. Burgdörfer, F. Krausz, and M. Drescher, Phys. Rev. Lett. 94, 023002 (2005); K. Ohmori, H. Katsuki, H. Chiba, M. Honda, Y. Hagihara, K. Fujiwara, Y. Sato, and K. Ueda, ibid. 96, 093002 (2006).

${ }^{5}$ M. V. Ammosov, N. B. Delone, and V. P. Krainov, Sov. Phys. JETP 64 1191 (1986)

${ }^{6}$ A. Becker and F. H. M. Faisal, J. Phys. B 38, R1 (2005).

${ }^{7}$ P. G. Burke and V. M. Burke, J. Phys. B 30, L383 (1997)

${ }^{8}$ S. I. Chu and D. A. Telnov, Phys. Rep. 390, 1 (2004).

${ }^{9}$ M. A. L. Marques and E. K. U. Gross, Annu. Rev. Phys. Chem. 55, 427 (2004).

${ }^{10}$ K. C. Kulander, Phys. Rev. A 35, 445 (1987).

${ }^{11}$ H. G. Muller, Laser Phys. 9, 138 (1999); D. Bauer and P. Koval, Comput. Phys. Commun. 174, 396 (2006).

${ }^{12}$ E. S. Smyth, J. S. Parker, and K. T. Taylor, Comput. Phys. Commun. 114, 1 (1998); K. T. Taylor, J. S. Parker, D. Dundas, K. J. Meharg, L.-Y Peng, B. J. S. Doherty, and J. F. McCann, Phys. Scr., T 110, 154 (2004).

${ }^{13}$ L.-Y. Peng, J. F. McCann, D. Dundas, K. T. Taylor, and I. D. Williams, J. Chem. Phys. 120, 10046 (2004).

${ }^{14}$ K. J. Meharg, J. S. Parker, and K. T. Taylor, J. Phys. B 38, 237 (2005).

${ }^{15}$ S. Qu and J. H. Eberly, Phys. Rev. A 44, 5997 (1991); M. S. Pindzola, P. Gavras, and T. W. Gorczyca, ibid. 51, 3999 (1995); V. Véniard, R. Taïeb, and A. Maquet, ibid. 60, 3952 (1999); C. Ruiz, L. Plaja, and L. Roso, Phys. Rev. Lett. 94, 063002 (2005).

${ }^{16}$ J. von Milczewski, D. Farrelly, and T. Uzer, Phys. Rev. Lett. 78, 2349 (1997); A. Gordon, R. Santra, and F. X. Kärtner, Phys. Rev. A 72 063411 (2005)

${ }^{17}$ G. L. Ver Steeg, K. Bartschat, and I. Bray, J. Phys. B 36, 3325 (2003).

${ }^{18}$ (a) B. I. Schneider and N. Nygaard, Phys. Rev. E 70, 056706 (2004); (b)
H. Kono, A. Kita, Y. Ohtsuki, and Y. Fujimura, J. Comput. Phys. 130, 148 (1997); (c) G. Czako, V. Szalay, A. G. Császár, and T. Furtenbacher, J. Chem. Phys. 122, 024101 (2005).

${ }^{19}$ D. Comtois, D. Zeidler, H. Pépin, J. C. Kieffer, D. M. Villeneuve, and P. B. Corkum, J. Phys. B 38, 1923 (2005); A. Rudenko, K. Zrost, Th. Ergler, A. B. Voitkiv, B. Najjari, V. L. B. de Jesus, B. Feuerstein, C. D. Schröter, R. Moshammer, and J. Ullrich, ibid. 38, L191 (2005).

${ }^{20}$ D. O. Harris, G. G. Engerholm, and W. D. Gwinn, J. Chem. Phys. 43, 1515 (1965)

${ }^{21}$ A. S. Dickinson and P. R. Certain, J. Chem. Phys. 49, 4209 (1968).

${ }^{22}$ J. V. Lill, G. A. Parker, and J. C. Light, Chem. Phys. Lett. 89, 483 (1982); R. W. Heather and J. C. Light, J. Chem. Phys. 79, 147 (1983); J. C. Light, I. P. Hamilton, and J. V. Lill, ibid. 82, 1400 (1985).

${ }^{23}$ J. C. Light and T. Carrington, Jr., Adv. Chem. Phys. 114, 263 (2000); H. S. Lee and J. C. Light, J. Chem. Phys. 120, 4626 (2004); J. Tennyson, M. A. Kostin, P. Barletta, G. J. Harris, O. L. Polyansky, J. Ramanlal, and N. F. Zobov, Comput. Phys. Commun. 163, 85 (2004).

${ }^{24}$ D. T. Colbert and W. H. Miller, J. Chem. Phys. 96, 1982 (1992); J. Echave and D. C. Clary, Chem. Phys. Lett. 190, 225 (1992); R. G. Littlejohn, M. Cargo, T. Carrington, Jr., K. A. Mitchell, and B. Poirier, J. Chem. Phys. 116, 8691 (2002); B. I. Schneider, Phys. Rev. A 55, 3417 (1997); T. N. Rescigno, D. A. Horner, F. L. Yip, and C. W. McCurdy, ibid. 72, 052709 (2005); B. I. Schneider and L. A. Collins, J. Non-Cryst. Solids 351, 1551 (2005).

${ }^{25}$ D. Baye and P. H. Heenen, J. Phys. A 19, 2041 (1986); V. Szalay, J. Chem. Phys. 99, 1978 (1993).

${ }^{26}$ J. P. Boyd, Chebyshev and Fourier Spectral Methods, 2nd ed. (Dover, New York, 2000), Chap. 5.

${ }^{27}$ G. Szegö, Orthogonal Polynomials (American Mathematical Society, Providence, RI, 1939).

${ }^{28}$ B. D. Shizgal and H. Chen, J. Chem. Phys. 104, 4137 (1996).

${ }^{29}$ W. Gautschi, ACM Trans. Math. Softw. 25, 213 (1999).

${ }^{30}$ C. A. Weatherford, E. Red, and A. Wynn III, Int. J. Quantum Chem. 90, 1289 (2002).

${ }^{31}$ G. Machtoub and C. Zhang, Int. J. Theor. Phys. 41, 293 (2002).

${ }^{32}$ K. M. Dunseath, J. M. Launay, M. Terao-Dunseath, and L. Mouret, J. Phys. B 35, 3539 (2002).

${ }^{33}$ Handbook of Mathematical Functions, edited by M. Abramowitz and I. A. Stegun (Dover, New York, 1965).

${ }^{34}$ C. Schwartz, J. Math. Phys. 26, 411 (1985).

${ }^{35}$ S. E. Choi and J. C. Light, J. Chem. Phys. 92, 2129 (1990).

${ }^{36}$ L. Y. Peng, D. Dundas, J. F. McCann, K. T. Taylor, and I. D. Williams, J. Phys. B 36, L295 (2003).

${ }^{37}$ D. A. Varshalovich, A. N. Moskalev, and V. K. Khersonskii, Quantum Theory of Angular Momentum (World Scientific, Singapore, 1988).

${ }^{38}$ G. W. Wei, S. C. Althorpe, D. J. Kouri, and D. K. Hoffman, J. Chem. Phys. 108, 7065 (1998).

${ }^{39}$ R. Guantes and S. C. Farantos, J. Chem. Phys. 111, 10827 (1999).

${ }^{40}$ D. Dundas, J. F. McCann, J. S. Parker, and K. T. Taylor, J. Phys. B 33, 3261 (2000).

${ }^{41}$ A. R. Barnett, Comput. Phys. Commun. 27, 147 (1982)

${ }^{42}$ M. D. Feit and J. A. Fleck, Jr., J. Comput. Phys. 47, 412 (1983).

${ }^{43}$ H. Tal-Ezer and R. Kosloff, J. Chem. Phys. 81, 3967 (1984).

${ }^{44}$ H. X. Qiao, Q. Y. Cai, J. G. Rao, and B. W. Li, Phys. Rev. A 65, 063403 (2002).

${ }^{45}$ T. J. Park and J. C. Light, J. Chem. Phys. 85, 5870 (1986); C. S. Guiang and R. E. Wyatt, Int. J. Quantum Chem. 67, 273 (1998); M. Hochbruck and C. Lubich, SIAM (Soc. Ind. Appl. Math.) J. Numer. Anal. 34, 1911 (1997); M. Hochbruck, C. Lubich, and H. Selhofer, SIAM J. Sci. Comput. (USA) 19, 1552 (1998).

${ }^{46}$ C. Leforestier, R. H. Bisseling, C. Cerjan et al., J. Comput. Phys. 94, 59 (1991); T. N. Truong, J. J. Tanner, P. Bala, J. A. McCammon, D. J. Kouri, B. Lesyng, and D. K. Hoffman, J. Chem. Phys. 96, 2077 (1991); C. Moler and C. Van Loan, SIAM Rev. 45, 3 (2003); A. Castro, M. A. L. Marques, and A. Rubio, J. Chem. Phys. 121, 3425 (2004).

${ }^{47}$ Y. Saad, Iterative Methods for Sparse Linear Systems, 2nd ed. (SIAM, Philadelphia, 2003), Chap. 6.

${ }^{48}$ R. B. Sidje, ACM Trans. Math. Softw. 24, 130 (1998).

${ }^{49}$ U. V. Riss and H. D. Meyer, J. Phys. B 28, 1475 (1995); J. G. Muga, J. P. Palao, B. Navarro, and I. L. Egusquiza, Phys. Rep. 395, 357 (2004); R. Lefebvre, M. Sindelka, and N. Moiseyev, Phys. Rev. A 72, 052704 (2005); O. Shemer, D. Brisker, and N. Moiseyev, ibid. 71, 032716 (2005); S. Scheit, H. D. Mayer, N. Moiseyev, and L. S. Cederbaum, J. Chem. Phys. 124, 034102 (2006). 
${ }^{50}$ T. K. Kjeldsen and L. B. Madsen, Phys. Rev. A 71, 023411 (2005).

${ }^{51}$ E. Cormier and P. Lambropoulos, J. Phys. B 29, 1667 (1996)

${ }^{52}$ C. Laughlin and S. I. Chu, Phys. Rev. A 48, 4654 (1993).

${ }^{53}$ D. A. Telnov and S. I. Chu, J. Phys. B 28, 2407 (1995).

${ }^{54}$ S. I. Chu and J. Cooper, Phys. Rev. A 32, 2769 (1985).

${ }^{55}$ M. Pont, D. Proulx, and R. Shakeshaft, Phys. Rev. A 44, 4486 (1991).

${ }^{56}$ L. D. Landau and E. M. Lifshitz, Quantum Mechanics, 2nd ed. (Pergamon, Oxford, 1965).

${ }^{57}$ A. Scrinzi, Phys. Rev. A 61, 041402 (2000).

${ }^{58}$ S. Geltman, J. Phys. B 33, 4769 (2000); A. A. Kudrin and V. P. Krainov, Laser Phys. 13, 1024 (2003); G. V. Dunne and C. S. Gauthier, Phys. Rev. A 69, 053409 (2004); R. M. Cavalcantil, P. Giacconi, and R. Soldati, J.
Phys. A 36, 12065 (2003).

${ }^{59}$ P. Durand and I. Paidarova, Eur. Phys. J. D 26, 253 (2003).

${ }^{60}$ D. Dimitrovski, T. P. Grozdanov, E. A. Solov'ev, and J. S. Briggs, J. Phys. B 36, 1351 (2003)

${ }^{61}$ D. Bauer and P. Mulser, Phys. Rev. A 59, 569 (1999).

${ }^{62}$ D. A. Telnov and S. I. Chu, J. Phys. B 37, 1489 (2004).

${ }^{63}$ K. R. Lykke, K. K. Murray, and W. C. Lineberger, Phys. Rev. A 43, 6104 (1991).

${ }^{64}$ C. Haritos, T. Mercouris, and C. A. Nicolaides, Phys. Rev. A 63, 013410 (2000).

${ }^{65}$ D. A. Telnov and S. I. Chu, J. Phys. B 29, 4401 (1996).

${ }^{66}$ D. A. Telnov and S. I. Chu, J. Phys. B 59, 2864 (1999). 